\title{
BIOMASSA E ATIVIDADE MICROBIANA DE SOLO SOB APLICAÇÃO DE RESÍDUOS ORGÂNICOS
}

\section{MICROBIAL BIOMASS AND ACTIVITY OF SOIL UNDER APPLICATION OF ORGANIC WASTE}

\author{
Danielle Helena Müller \\ Universidade Federal de Mato Grosso \\ E-mail: dan.hm@hotmail.com \\ Elisangela Clarete Camili \\ Universidade Federal de Mato Grosso \\ E-mail: eccamili@hotmail.com \\ Sebatião Carneiro Guimarães \\ Universidade Federal de Mato Grosso \\ E-mail: sheepterra@terra.com.br \\ Daniela Tiago da Silva Campo \\ Universidade Federal de Mato Grosso \\ E-mail: camposdts@yahoo.com.br \\ Maíra de Emílio Martins \\ Universidade Federal de Mato Grosso \\ E-mail: maira.martins@sementesadriana.com.br \\ Karoline da Costa Barros \\ Universidade Federal de Mato Grosso \\ E-mail: karolinecbarros@gmail.com
}

\section{RESUMO}

O objetivo deste trabalho foi avaliar a influência de adubos orgânicos na população microbiana e sua atividade no solo. Para tal, foram coletadas, em dois períodos, amostras de solo na Fazenda Experimental da Universidade Federal de Mato Grosso, no Município de Santo Antônio de Leverger. O delineamento experimental adotado foi em blocos casualizados, com quatro repetições de quatro plantas cada. Retirou-se amostras de solo na profundidade de 0-20 $\mathrm{cm}$, de áreas adubadas comseis fontes de adubos orgânicos, aplicados em cobertura, nas quantidades determinadas pelo teor de $\mathrm{N}$ nos materiais orgânicos:esterco de bovinos, esterco de aves, efluente de suínos, composto de esterco de bovinos, composto de esterco de aves e composto de efluente de suínos. Avaliou-se o carbono da biomassa microbiana (CBM), a respiração microbiana $(\mathrm{RM})$ e o quociente metabólico $\left(\mathrm{qCO}_{2}\right)$.Os valores de carbono da biomassa microbiana dos estercos de aves e bovinos foram maiorese, portanto estimulam o aporte de microrganismos no solo. Os adubos orgânicos, exceto o esterco de aves curtido, não alteram a atividade dos microrganismos.

Palavras-chave: Biomassa Microbiana; Matéria Orgânica; Adubação Orgânica. 


\begin{abstract}
The aim of this study was to evaluate the influence of organic fertilizers on the microbial population and its activity in the soil. For this purpose, were collected during two periods, soil samples at the Experimental Farm of the Federal University of MatoGrosso, in Santo Antônio de Leverger. Samples were taken at depth of $0-20 \mathrm{~cm}$, areas fertilized with six sources of organic fertilizers applied in coverage, in amounts determined by the $\mathrm{N}$ content in organic materials: cattle manure, poultry manure, swine effluent consisting of cattle manure, poultry manure compost and compost wastewater pig. Evaluated the microbial biomass carbon (MBC), microbial respiration (MR) and metabolic quotient $\left(\mathrm{qCO}_{2}\right)$. Means were compared by the Scott Knott test at $5 \%$. The poultry and cattle manures have higher values of microbial biomass carbon, thus stimulate the contribution of microorganisms in the soil. Organic fertilizers, except poultry manure tanned, do not cause disturbances in the activity of microorganisms in periods.
\end{abstract}

Keywords: Microbial biomass; Organic Matter, Organic Fertilization.

\title{
INTRODUÇÃO
}

A adubação orgânica é uma prática agrícola muito utilizada para a melhoria das propriedadesdo solo (SEVERINOet al., 2006) e representa uma importante estratégia de manejo, (LEITEet al., 2003), influenciando positivamente as características biológicas do solo (Pires et al., 2008), ao favorecer inúmeros processos microbiológicos relacionados com a mineralização e a liberação de nutrientes às plantas (BENTO,1997).

A biomassa microbiana possui papel fundamental na produtividade e na manutenção do solo, pois atua como catalisadora das importantes transformações químicas e constitui reservatório de nutrientes disponíveis às plantas, por pertencer ao componente lábil da matéria orgânica do solo e possuir atividade influenciada pelas condições bióticas e abióticas (Monteiro e GAMA-RODRIGUES, 2004), assim é um indicador microbiano útil para avaliar alterações na propriedade biológica do solo (BAUHUSet al., 1998).

O objetivo do trabalho foi avaliar a influência de adubos orgânicos na população microbiana e sua atividade no solo.

\section{MATERIAL E MÉTODOS}

\section{Área de estudo}


O experimento foi realizado na Fazenda Experimental da Universidade Federal de Mato Grosso - UFMT, situada no município de Santo Antônio de Leverger/MT ( $15^{\circ} 46^{\prime} \mathrm{S}, 56^{\circ} 05^{\prime} \mathrm{W}$, altitude de 104 metros). O clima é do tipo Aw, segundo a classificação de Köppen, caracterizando-se por um período seco de maio a setembro e outro chuvoso que se estende de outubro a abril. A temperatura média anual foi de $25,6^{\circ} \mathrm{C}$ e precipitação anual de $1.421 \mathrm{~mm}$. O solo predominante é caracterizado como Latossolo Amarelo Eutrófico típico de textura média (EMBRAPA, 2006).

\section{Delineamento experimental}

O delineamento experimental adotado foi em blocos ao acaso, com quatro repetições, e os tratamentos foram constituídos por seis diferentes fontes de adubos orgânicos, aplicados em cobertura parcelada, nas quantidades determinadas pelo teor de $\mathrm{N}$ nos materiais orgânicos, sendo $25 \%$ no mês de julho, $25 \%$ no mês de setembro, $25 \%$ no mês de novembro de 2010 e $25 \%$ no mês de janeiro de 2011 . O solo foi coletado em dois períodos:

Tratamento 1 - solo adubado com esterco de bovinos curtido, coletado 60 dias após a segunda adubação;

Tratamento 2 -solo adubado com esterco de aves curtido, coletado 60 dias após a segunda adubação;

Tratamento 3 - solo adubado com efluente de suínos (líquido), coletado 60 dias após a segunda adubação;

Tratamento 4 -solo adubado com composto de esterco de bovinos, coletado 60 dias após a quarta adubação;

Tratamento 5 - solo adubado com composto de esterco de aves, coletado 60 dias após a quarta adubação;

Tratamento 6 -solo adubado com composto de efluente de suínos, coletado 60 dias após a quarta adubação.

Os compostos utilizados na adubaçãoapresentaram-se prontos aos 75 dias, foram analisados e os resultados são apresentados na Tabela 1.

\section{Análises Laboratoriais}

A influência da adubação orgânica sobre os atributos microbiológicos do solo foi avaliada determinando-se os teores de carbono presente na biomassa 
microbiana, e estimando-se a respiração microbiana e o quociente metabólico do solo.

Tabela 1. Composição química dos estercos e compostos utilizados como adubação de cobertura. Santo Antônio de Leverger/MT. Julho, 2010.

\begin{tabular}{llllllllll}
\hline $\begin{array}{l}\text { Relação } \\
\mathrm{C} / \mathrm{N}\end{array}$ & $\mathrm{pH}$ & Umidade & $\mathrm{MO}$ & $\mathrm{C}$ & $\mathrm{N}$ & $\mathrm{P}_{2} \mathrm{O}_{5}$ & $\mathrm{~K}_{2} \mathrm{O}$ & $\mathrm{Ca}$ & $\mathrm{Mg}$ \\
\hline
\end{tabular}

\begin{tabular}{|c|c|c|c|c|c|c|c|c|c|c|}
\hline $\mathrm{T} 1$ & 7,16 & 6,6 & 23 & 10 & 5,61 & 0,78 & 0,68 & 0,36 & 0,06 & 0,25 \\
\hline T2 & 7,67 & 7,6 & 29 & 21 & 12,25 & 1,60 & 3,63 & 2,22 & 5,60 & 0,66 \\
\hline T3 & 0,38 & 8,0 & 86 & 02 & 1,13 & 0,29 & 0,15 & 0,05 & 0,00 & 0,04 \\
\hline T4 & 7,41 & 6,7 & 36 & 09 & 5,19 & 0,70 & 0,62 & 0,27 & 0,50 & 0,21 \\
\hline T5 & 3,83 & 7,4 & 50 & 12 & 6,87 & 1,79 & 2,31 & 1,84 & 6,00 & 0,33 \\
\hline T6 & 1,50 & 5,6 & 71 & 06 & 3,36 & 2,24 & 0,58 & 0,10 & 0,54 & 0,27 \\
\hline
\end{tabular}

As amostras de solo para análise foram coletadas na camada de 0 a 20 $\mathrm{cm}$ de profundidade, retirando-se de cada parcela uma amostra composta por duas subamostras, que foram acondicionadas e transportadas para 0 laboratório e permaneceram em câmara fria a $4^{\circ} \mathrm{C}$ até o momento das análises. Retirou-se 20 gde cada amostra do solo coletadopara determinação do grau de umidade, por secagemem estufa a $105^{\circ} \mathrm{C}$, até o peso constante.

\section{Carbono da Biomassa Microbiana do solo}

A análise do CBM foi realizada segundo método clorofórmio fumigação incubação (CFI) proposto por Jenkinson\&Polwson (1976).

De cada amostra do solo, foram retiradas duas subamostras. Uma delas, pesando $50 \mathrm{~g}$, foi colocada em frasco de vidro com tampahermética com capacidade de $800 \mathrm{~mL}$. A segunda amostra, pesando $48 \mathrm{~g}$, foi colocada em béquer de $200 \mathrm{~mL}$ e fumigada em dessecador acoplado na bomba a vácuo, contendo um béquer de $50 \mathrm{~mL}$ contendo $20 \mathrm{~mL}$ de clorofórmio livre de álcool. As paredes do dessecador foram forradas com papel úmido para o solo não 
perder umidade. As amostras foram mantidas no dessecador por $24 \mathrm{~h}$, no escuro e em temperatura ambiente.

Após o período de fumigação, o vapor de clorofórmio do dessecador e das amostras foi evacuado, sucessivas vezes, com auxílio da bomba a vácuo.

As amostras de solo fumigadas foram transferidas para o frascos de vidro com tampa hermética e reinoculados com $2 \mathrm{~g}$ de solo da mesma amostra original. Em cada vidro, das amostras fumigadas e não fumigadas, foi colocado um copo plástico de $80 \mathrm{~mL}$ contendo $20 \mathrm{~mL}$ deNaOH 0,5M. As amostras foram tampadas hermeticamente e mantidas no escuro por dez dias à temperatura ambiente.

A quantidade de $\mathrm{CO}_{2}$ liberada do solo foi determinada após a adição de $3 \mathrm{~mL}$ de $\mathrm{BaCL}_{2} 30 \%$ e posterior titulação com $\mathrm{HCl}$ 0,3M da solução deNaOH 0,5M usando fenolftaleína 1\% como indicador. Foram utilizados como controle três recipientes de vidro, sem solo, contendo a mesma solução de $\mathrm{NaOH}$ 0,5M.

O carbono da biomassa microbiana foi calculado pela diferença entre 0 $\mathrm{CO}_{2}$ liberado das amostras fumigadas (F) e amostras não fumigadas (NF), utilizando o fator de correção (Kc) de 0,45 (ANDERSON; DOMSCH, 1978). Os dados de carbono da biomassa microbiana foram expressos em $\mathrm{mg} \mathrm{C} \mathrm{kg}^{-1}$ solo.

\section{Respiração microbiana}

A avaliação da RM foi realizada juntamente com a avaliação do CBM, sendo estimada pela quantidade de $\mathrm{CO}_{2}$ liberado do solo não fumigado, durante dez dias de incubação. Os dados da respiração microbiana foram expressos em mg $\mathrm{C} \mathrm{kg}^{-1}$ solo.

\section{Quociente metabólico}

O quociente metabólico $\left(q \mathrm{CO}_{2}\right)$ foi calculadopela razão entre a taxa de RM e o CBM(ANDERSON; DOMSCH, 1993), sendo expressoem mg C-CO $\mathrm{CO}_{2} \mathrm{mg}$ $\mathrm{Cmic}^{-1} \mathrm{dia}^{-1}$, conforme a fórmulaa seguir:

$$
\begin{aligned}
& \mathrm{CO}_{2}=\mathrm{TRM} / \mathrm{CBM}(1) \\
& \mathrm{TRM}=\mathrm{RM} / \mathrm{d}(2)
\end{aligned}
$$

em que:

$\mathrm{qCO}_{2}=$ quociente metabólico ( $\mathrm{mg} \mathrm{C}-\mathrm{CO}_{2} \mathrm{mgCmic}^{-1} \mathrm{dia}^{-1}$ ); 
$\mathrm{TRM}=$ taxa de respiração microbiana $\left(\mathrm{mg} \mathrm{C}-\mathrm{CO}_{2} \mathrm{~kg}^{-1} \mathrm{dia}^{-1}\right)$;

$\mathrm{RM}=$ respiração microbiana $\left(\mathrm{mg} \mathrm{C}-\mathrm{CO}_{2} \mathrm{~kg}^{-1}\right)$;

$\mathrm{d}=$ dias de incubação para a determinação darespiração microbiana;

$\mathrm{CBM}=$ carbono da biomassa microbiana $\left(\mathrm{mg} \mathrm{C} \mathrm{kg}^{-1}\right)$.

\section{Análise estatística}

O delineamento utilizado foi em fatorial $6 \times 2$, sendo seis fontes de adubos orgânicos e dois períodos de coleta. Os dadosobtidos foram submetidos à análise de variância e asmédias comparadas pelo teste de ScottKnott a $5 \%$.

\section{RESULTADOS E DISCUSSÃO}

Os diferentes adubos orgânicos (tratamentos) influenciaram O CBM (Tabela 2). Os valores do CBM desprendidos em dez dias de incubação, variaram de 8,90 a 40,60 $\mathrm{mg} \mathrm{C} \mathrm{kg}^{-1}$ de solo,ambos na segunda coleta.

Tabela 2. Carbono da biomassa microbiana (CBM) em solo adubado com diferentes fontes orgânicas. Santo Antônio de Leverger/MT, 2010.

\begin{tabular}{lcc}
\hline & Primeira coleta & Segunda coleta \\
\cline { 2 - 3 } Tratamentos & \multicolumn{2}{c}{ CBM } \\
\cline { 2 - 3 } & $16,86 \mathrm{mg}$ C mic g \\
Esterco de bovinos & $23,06 \mathrm{aA}$ & $40,60 \mathrm{aA}$ \\
Esterco de aves & $9,63 \mathrm{cA}$ & $8,90 \mathrm{~dB}$ \\
Efluente de suínos & $12,31 \mathrm{cB}$ & $11,11 \mathrm{dA}$ \\
Composto de esterco de bovinos & $9,46 \mathrm{cB}$ & $19,64 \mathrm{bA}$ \\
Composto de esterco de aves & $19,24 \mathrm{bA}$ & $14,00 \mathrm{cA}$ \\
Composto de efluente de suínos & $14,13 \mathrm{cB}$ \\
\hline * Médias seguidas pela mesma letra minúscula nas colunas e maiúsculas nas linhas não \\
diferem entre si pelo teste de Scott-Knott a 5\% de probabilidade.
\end{tabular}

$\mathrm{Na}$ avaliação do CBM na primeira coleta, realizada 60 dias após a segunda aplicação dos adubos, o esterco de aves (tratamento 2) obteve maior valor, 23,06 mg C mic g ${ }^{-1}$ solo (Tabela 2). Considerando que o crescimento microbiano é limitado, muitas vezes pela escassez de nutrientes encontrados no solo, a adição de fontes de carbono ou nitrogênio pode aumentar a biomassa (GRAHAMet al., 2002). Dessa forma, valores elevados de CBM 
implicam em maior imobilização temporária de nutrientes e, consequentemente, menor propensão a perdas dos mesmos no sistema soloplanta (MERCANTEet al., 2004).

Segundo Leite et al. (2003) os resíduos orgânicos depositados no solo, após a decomposição, são essenciais no processo de adição de carbono orgânico ao longo do tempo, sendo importantes para a sustentabilidade do sistema, pela influência da matéria orgânica nas propriedades físicas, químicas e biológicas do solo.

O esterco de aves (tratamento 2) apresentou maior conteúdo de CBM na primeira coleta, sendo superior $(p<0,05)$ aos demais tratamentos. Por outro lado, foi o tratamento, seguido pelo efluente de suínos (tratamento 3), que apresentou menor valor de CBM na segunda coleta (Tabela 2). Esse fato ocorreu, provavelmente, em função da grande quantidade de matéria orgânica adicionada ao solo em curto espaço de tempo, dificultando a ação dos microorganismos em decompô-la.

$\mathrm{Na}$ avaliação da respiração microbiana, não observou-sediferença entre os adubos aplicados em cobertura. Contudo verificou-se acréscimo nos valores da respiração dos microrganismosnos diferentes períodos de coleta para todosos tratamentos (Tabela 3).

Tabela 3. Respiração microbiana (RM) em solo adubado com diferentes fontes orgânicas. Santo Antônio de Leverger/MT, 2010.

\begin{tabular}{lcc}
\hline & Primeira coleta & \multicolumn{2}{c}{ Segunda coleta } \\
\hline Tratamentos & \multicolumn{2}{c}{$\mathrm{RM}$} \\
\cline { 2 - 3 } Esterco de bovinos & $0,78^{\text {ns }} \mathrm{CO}_{2} \mathrm{~g}^{-1}$ & solo \\
Esterco de aves & 0,66 & 1,54 \\
Efluente de suínos & 0,48 & 0,92 \\
Composto de esterco de bovinos & 1,45 & 0,52 \\
Composto de esterco de aves & 0,79 & 1,55 \\
Composto de efluente de suínos & 1,22 & 1,22 \\
\hline
\end{tabular}

* NS - não significativo

Considerando a mesma constituição da comunidade microbiana, a biomassa microbiana do solo "eficiente" teria menor taxa de respiração. Porém, Roscoeet al. (2006) afirmaram que a alta taxa de respiração pode ser interpretada como característica desejável quando se considera que a 
decomposição dos resíduos orgânicos irá disponibilizar nutrientes para a planta.

Assim, Islam\&Weil (2000) concluiram que a taxa de respiração mais elevada pode ser desejável ou não, podendo indicar tanto distúrbio como alto nível de produtividade do ecossistema, devendo ser analisada em cada contexto.

A avaliação da biomassa e respiração microbiana feita isoladamente pode frequentemente, fornecer apenas informações limitadas sobre as respostas do sistema solo a estresse ou perturbações. Outras avaliações, portanto, podem ser conduzidas juntamente com a determinação dessas características, como o quociente metabólico. A taxa de respiração por unidade de biomassa microbiana $\left(\mathrm{qCO}_{2}\right)$, apresenta-se como variável de determinação mais adequada para o entendimento (ALVESet al. 2011).

$\mathrm{O}$ quociente metabólico, expresso em quantidade de $\mathrm{CO}_{2}$ liberado pela quantidade de carbono da biomassa em função do tempo, representa a taxa de respiração específica da biomassa microbiana. Altos valores do $\mathrm{qCO}_{2}$ significam que a população microbiana está oxidando carbono de suas próprias células (respiração de manutenção dos microrganismos vivos) para a sua manutenção e adaptação ao solo, portanto, a população microbiana se encontra em condições adversas ou estressantes (ANDERSON; DOMSCH, 1993; ISLAM; WEIL, 2000).

O maior valor de quociente metabólico foi observado ao se aplicar os compostos de estercos de bovinos e aves e o composto de efluente de suínos (tratamentos 4, 5 e 6, respectivamente) na primeira coleta e indicam possível estresse sobre os microrganismos (Tabela 4).

Tabela 4. Quociente metabólico $\left(q \mathrm{CO}_{2}\right)$ em solo adubado com diferentes fontes orgânicas. Santo Antônio de Leverger/MT, 2010.

\begin{tabular}{lcc}
\hline & \multicolumn{2}{c}{ Primeira coleta } \\
\hline Tratamentos & \multicolumn{2}{c}{$\mathrm{qCO}_{2}$} \\
\cline { 2 - 3 } Esterco de bovinos & \multicolumn{2}{c}{${\mathrm{mg} \mathrm{C}-\mathrm{CO}_{2} \mathrm{mg}^{-1} \mathrm{C} \mathrm{mic} \mathrm{dia}}^{-1}$} \\
Esterco de aves & $0,05 \mathrm{bA}^{*}$ & $0,03 \mathrm{aA}$ \\
Efluente de suínos & $0,02 \mathrm{bB}$ & $0,10 \mathrm{aA}$ \\
Composto de esterco de bovinos & $0,05 \mathrm{bA}$ & $0,04 \mathrm{aA}$ \\
Composto de esterco de aves & $0,12 \mathrm{aA}$ & $0,08 \mathrm{aA}$ \\
Composto de efluente de suínos & $0,09 \mathrm{aA}$ & $0,09 \mathrm{aA}$ \\
\hline
\end{tabular}


* Médias seguidas pela mesma letra minúscula nas colunas e maiúsculas nas linhas não diferem entre si pelo teste de Scott-Knott a $5 \%$ de probabilidade.

Este comportamento pode ser explicado pela qualidade da matéria orgânica empregada, considerando que nos estercos de bovinos e aves e efluente de suínos (tratamentos 1, 2 e 3, respectivamente), provavelmente os adubos estavam mais prontamente disponíveis ao ataque e fácil decomposição pelos microrganismos, o que pode ter reduzido o gasto energético (Tabela 4).

A decomposição mais lenta dos resíduos, devido principalmente à relação C/N dos materiais (ALVARENGA, 1993), ocasiona distúrbios na comunidade microbiana do solo e o reparo de danos requer desvio de energia do crescimento e reprodução para a manutenção celular, de forma que uma porção do carbono da biomassa será perdida como $\mathrm{CO}_{2}$ (SAMPAIOet al., 2008).

Avaliando-se os adubos nos diferentes períodos de coleta, observa-se valor superior, para o esterco de aves (tratamento 2), na segunda coleta. Este fator pode estar relacionado ao acréscimo considerável deste adubo, que em comparação aos demais apresenta maior quantidade de teor de matéria orgânica $(21 \%)$ e carbono (12,25\%), que pode ao longo do tempo se acumular, pela menor taxa de decomposição, aumento assim a atividade dos microrganismos.

Souza et al. (2006) observaram que a aplicação de vermicomposto originado de esterco de codornas em Latossolo Vermelho sob Cerrado, submetido à diferentes sistemas de manejo e usos do solo, aumentou os teores de carbono orgânico total e promoveu maior atividade microbiana, evidenciada pelos aumentos na taxa de respiração microbiana e no quociente metabólico do solo.

Os valores de quociente metabólico inferiores a 1,0 em todos os tratamentos, para as diferentes coletas,sugerem disponibilidade da matéria orgânica para os microrganismos (SAMPAIOet al., 2008).

Admite-se que valores inferiores de quociente metabólico indicam que os microorganismos estão sob menor nível de estresse e a menor relação de respiração por unidade de carbono da biomassa microbiana é frequente em sistemas mais estáveis para diferentes espécies vegetais (VICENZlet al., 2002). 
Considerando que as propriedades biológicas do solo têm relação direta com a presença de material orgânico disponível, refletindo mudanças na concentração do conteúdo de matéria orgânica do solo (REZENDEet al., 2004), é possível através deste trabalho, verificar que a aplicação dos adubos orgânicos ocasionaram aporte nos parâmetros microbiológicos, beneficiando o sistema solo-planta.

\section{CONCLUSÕES}

Os valores de carbono da biomassa microbiana são maiores nos estercos de aves e bovinos.

Os adubos orgânicos, exceto o esterco de aves curtido, não alteram a atividade dos microrganismos nos períodos avaliados.

\section{REFERÊNCIAS BIBLIOGRÁFICAS}

ALVARENGA, R. C. Potencialidades de adubos verdes para conservação e recuperação de solos. Viçosa : UFV, 1993. 112p. (Tese de Doutorado).

ALVES, T. S.; LIZIA LENZA CAMPOS, L. L.; , ELIAS NETO, N.; MATSUOKA, M.; LOUREIRO, M. F. Biomassa e atividade microbiana de solo sob vegetação nativa e diferentes sistemas de manejos. Acta ScientiarumAgronomy, v. 33, n. 2, p. 341-347, 2011.Disponível em: <www.scielo.br/pdf/asagr/v33n2/21.pdf>.

ANDERSON, J. P. E.; DOMSCH, K. H.A physiological method for the quantitative measurement of microbial biomass in soils.Soil Biology and Biochemistry, v. 10, n. 3, p. 215-221, 1978.Disponívelem: <http://www.sciencedirect.com/science/article/pii/0038071778900998>.

ANDERSON, J. P. E.; DOMSCH, K. H.The metabolic quotient of $\mathrm{CO} 2$ (q CO2) as a specific activity paramenter to assess the effects of environmental condition, such as $\mathrm{pH}$, on the microbial of forest soil.Soil Biology andBiochemistry, v. $25, \quad$ n. $3, \quad$ p. 393-395, 1993.Disponívelem: $<w w w . s c i e n c e d i r e c t . c o m / s c i e n c e / a r t i c l e / p i i / 0038071793901407>$.

BAUHUS, J.; PARÉ, D.; COTÉ, L. Effects of tree species, stand age and soil type on soil microbial biomass and its activity in a southern boreal forest. SoilBiology and Biochemistry, v. 30, p. 1077-1089, 1998.Disponívelem: <www.sciencedirect.com/science/article/pii/S0038071797002137>.

BENTO, M. M. Fontes de Matéria orgânica na composição de substratos para a produção de mudas micorrizas de maracujazeiro. 1997. 59p. Dissertaçãode Mestrado- ESALQ. Piracicaba-SP, 1997. 
CATTELAN, A. J.; VIDOR, C. Flutuações na biomassa, atividade e população microbiana do solo em função de variações ambientais. Revista Brasileira deCiência do Solo, v. 14, n. 2, p. 133-142, 1990.

EMPRESA BRASILEIRA DE PESQUISA AGROPECUÁRIA-EMBRAPA. Sistema brasileiro de classificação de solos. 2 ed. Rio de Janeiro: Embrapa Solos. 2006. 306 p.

ESPINDOLA, J. A. A.; GUERRA, J. G. M.; ALMEIDA, D. L.; TEIXEIRA, M. G.; URQUIAGA, S. Decomposição e liberação de nutrientes acumulados em leguminosas herbáceas perenes consorciadas com bananeiras. Revista Brasileira de Ciência do Solo, v. 30, p. 321-328, 2006.Disponível em: <http://www.scielo.br/pdf/rbcs/v30n2/a12v30n2.pdf>.

GRAHAM, M. H.; HAYNES, R. J.; MEYER, J. H. Soil organic matter content and quality: effects of fertilizer applications, burning and trash retention on a long-term sugarcane experiment in South Africa. Soil Biology and Biochemistry, v. 34, p. 93-102, 2002.Disponívelem: $<w w w . s c i e n c e d i r e c t . c o m / s c i e n c e / a r t i c l e / p i i / S 0038071701001602>$.

ISLAM, K. R.; WEIL, R. R. Land use effects on soil quality in a tropical forest ecosystem of Bangladesh.Agriculture Ecosystems and Environment, v. 79, n. $1, \quad$ p. $\quad 9-16, \quad$ 2000.Disponívelem: <www.sciencedirect.com/science/article/pii/S0167880999001450>.

JENKINSON, D. S.; POWLSON, D. S.The effects of biocidal treatments on metabolism in soil. V. A method for measuring soil biomass. Soil Biology and Biochemistry, v. 8, p. 209-213, 1976.Disponível em: $<w w w . s c i e n c e d i r e c t . c o m / s c i e n c e / a r t i c l e / p i i / 0038071776900055>$.

LEITE, L. F. C.; MENDONÇA, E. S.; NEVES, J. C. L.; MACHADO, P. L. O. A.; GALVÃO, J. C. C. Estoques totais de carbono orgânico e seus compartimentos em Argissolosob floresta e sob milho cultivado com adubação mineral e orgânica. Revista Brasileira de Ciência do Solo, v. 27, p. 821-832, 2003.Disponível em: <www.scielo.br/pdf/rbcs/v27n5/v27n5a06.pdf>.

MERCANTE, F. M.; FABRICIO, A. C.; MACHADO, L. A. Z.; SILVA, W. M. Parâmetros microbiológicoscomo indicadores de qualidade do solo sobsistemas integrados de produção agropecuária. Dourados: Embrapa Agropecuária Oeste, 2004.

MONTEIRO, M. T.; GAMA-RODRIGUES, E. F. Carbono, nitrogênio e atividade da biomassa microbiana em diferentes estruturas de serapilheira de uma floresta natural. Revista Brasileira de Ciência do Solo, v. 28, p. 819-826, 2004.Disponível em: <www.scielo.br/pdf/rbcs/v28n5/22817.pdf>.

PIRES, A. A.; MONNERAT, H. P.; MARCIANO, C. R.; PINHO, L. G. R.; ZAMPIROLLI, P. D.; ROSA, R. C.; MUNIZ, R. A. Efeito da adubação alternativa do maracujazeiro amarelo nas características químicas e físicas do solo. Revista Brasileira de Ciência do Solo, v. 32, p. 1997-2005, 2008.Disponível em: <www.scielo.br/pdf/rbcs/v32n5/21.pdf>. 
REZENDE, L. A.; ASSIS, L. C.; NAHAS, E. Carbon, nitrogen and phosphorous mineralization in two soils amended with distillery yeast. Bioresource Technology, v. 94, p. 159-167, 2004.Disponível em: $<w w w . s c i e n c e d i r e c t . c o m / s c i e n c e / a r t i c l e / p i i / S 0960852403003699>$.

ROSCOE, R.; MERCANTE, F. M.; SALTON, J. C. Dinâmica da matéria orgânica do solo em sistemas conservacionistas: modelagem matemática e métodos auxiliares. Dourados: Embrapa Agropecuária Oeste, 2006.

SAMPAIO, D. B.; ARAÚJO, A. S. F.; SANTOS, V. B. Avaliação de indicadores biológicos de qualidade do solo sob sistemas de cultivo convencional e orgânico de frutas. Ciência e Agrotecnologia, v. 32, n. 2, p. 353-359, 2008.Disponível em: <www.scielo.br/pdf/cagro/v32n2/01.pdf>.

SEVERINO, L. S.; FERREIRA, G. B.; MORAES, C. R. A.; GONDIM, T. M. S.; CARDOSO, G. D.; VIRIATO, J. R.; BELTRÃO, N. E. M. Produtividade e crescimento da mamoneira em resposta à adubação orgânica e mineral. Pesquisa Agropecuária Brasileira, v. 41, n. 5, p. 879-882, 2006.Disponível em: <www.scielo.br/pdf/pab/v41n5/30612.pdf>.

SOUZA, E. D.; CARNEIRO, M. A. C.; PAULINO, H. B.; SILVA, C. A. Frações do carbono orgânico, biomassa e atividade microbiana em um Latossolo Vermelho sob cerrado submetido a diferentes sistemas de manejos e usos do solo. Acta ScientiarumAgronomy, v. 28, n. 3, p. 323-329, 2006.Disponível em: <periodicos.uem.br/ojs/index.php/ActaSciAgron/article/viewFile/940/469>.

TORRES, J. L. R.; PEREIRA, M. G.; FABIAN, A. J.; PAULA, J. C. Efeito da temperatura do ar e precipitação pluviométrica na decomposição de plantas de cobertura no Cerrado. In: CONGRESSO BRASILEIRO DA CIÉNCIA DO SOLO, 31. Gramado, 2007. Anais...Gramado, SBCS/UFRGS, 2007.

VASCONCELLOS, C. A. Temperature effect on carbon biomass in soils from tropical and temperate regions, Scientia Agricola, v. 55, n. 1, p. 94-104, 1998.Disponível em: <www.scielo.br/scielo.php?script=sci_arttext\&pid=S010390161998000100016>.

VICENZI, M.; FACHINELLO, J. C.; CASTILHOS, D. D.; SANTOS, V. B.; SILVA, D. G. Biomassa e atividade microbiana em sistemas de produção integrada (PI) e convencional (PC) na cultura do pêssego In: XXV REUNIÃO BRASILEIRA DE FERTILIDADE DO SOLO E NUTRIÇÃO DE PLANTAS. Rio de Janeiro. Anais...Rio de janeiro: SBCS, 2002. 